\title{
SOX10 is abnormally expressed in aganglionic bowel of Hirschsprung's disease infants
}

\author{
M H Sham, V C H Lui, M Fu, B Chen, P K H Tam
}

\begin{abstract}
Background-The primary pathology of Hirschsprung's disease (HD) is a congenital absence of ganglion cells in the caudal most gut. The spastic aganglionic bowel is often innervated by a network of hypertrophied nerve fibres. Recently, mutations of SOX10 have been identified in patients with HD but only in those with Waardenburg-Shah syndrome.

Aims-To understand the molecular basis for the pathogenesis of HD we intended to determine the specific cell lineages in the enteric nervous system which normally express $S O X 10$ but are affected in disease conditions.
\end{abstract}

Methods-We studied colon biopsies from 10 non-syndromic HD patients, aged three months to four years, and 10 age matched patients without HD as normal controls. The absence of mutation in the SOX10 gene of HD patients was confirmed by DNA sequencing. Expression and cellular distribution of SOX10 in bowel segments of normal and HD infants were examined by reverse transcriptionpolymerase chain reaction and in situ hybridisation.

Results-We found that in normal infants and normoganglionic bowel segments of HD patients, SOX10 was expressed in both neurones and glia of the enteric plexuses and in the nerves among the musculature in normal colon. In the aganglionic bowel segments of patients, SOX10 expression was consistently lower and was found to be associated with the hypertrophic nerve trunks in the muscle and extrinsic nerves in the serosa.

Conclusion-We conclude that SOX10 is normally required postnatally in the functional maintenance of the entire enteric nervous system, including neurones and glia. In non-syndromic HD patients who do not have the SOX10 mutation, the SOX10 gene expressed in the sacral region may be involved in the pathogenesis of the abnormal nerve trunks through interaction with other factors.

(Gut 2001;49:220-226)

Keywords: SOX10; polymorphism; enteric nervous system; Hirschsprung's disease; colon; neurocristopathy

rofessor P K H Tam Division of Paediatric Surgery, Department of Surgery, University of Hong Kong Medical Centre, Queen Mary Hospital,

Pokfulam, Hong Kong SAR, China.

paultam@hkucc.hku.hk

Accepted for publication 29 January 2001

The enteric nervous system (ENS) is a unique division of the nervous system which controls important gut functions, including motility, and can function independently of the central nervous system. It consists of a large number of neurones and specialised enteric glia aggregated in interconnecting ganglia in plexuses within the gut wall. The ENS arises from neural crest cells through a complex process of migration, proliferation, growth, and differentiation. Interference with normal development of the ENS results in neurocristopathies, such as Hirschsprung's disease (HD) which occurs in 1:5000 births. The primary pathology of HD is a congenital absence of ganglion cells in the distal gut which leads to intestinal obstruction, resulting in considerable morbidity and mortality.

HD is a polygenic disease. About half of familial and 17-20\% of sporadic cases are associated with the RET receptor tyrosine kinase gene, ${ }^{1-6}$ and fewer with mutations in the endothelin 3 (EDN3), ${ }^{78}$ endothelin $\mathrm{B}$ receptor $(E D N R B),{ }^{9-13}$ glial derived neurotrophic factor $(G D N F),{ }^{5}$ neuturin, ${ }^{14}$ and unidentified modifier $^{15}$ genes. Recently, mutations in the SOX10 gene have been described in HD patients but only in those who also show pigmentary defects and/or deafness (Waardenburg-Shah syndrome). ${ }^{16-19}$ The SOX10 gene encodes a HMG domain containing Sry related transcription factor. ${ }^{20}$ The presence of SOX10 mutations in Waardenburg-Hirschsprung patients suggests that the SOX10 gene could be involved in regulatory and signalling pathways for the early development of the neural crest cell lineages which differentiate into melanocytes and enteric ganglia. The involvement of Sox10 in the development of enteric neurones has also been demonstrated in the Dom (Dominant megacolon) mouse model of HD. It was shown that a single base insertion in the mouse Sox 10 gene was responsible for the megacolon phenotype of the Dom mutant. ${ }^{2122}$

The ENS mainly originates from the vagal neural crest during early development but there is also a small contribution of enteric ganglia from the sacral neural crest in the distal gut. ${ }^{23-27}$ In the aganglionic colon of HD, the sacral neural crest is unable to compensate for the loss of vagal neural crest derived enteric ganglia. ${ }^{28-30}$ Moreover, the spastic aganglionic bowel is often innervated by a network of hypertrophied nerve fibres which are thought to be extrinsic preganglionic parasympathetic nerve fibres originating from the sacral parasympathetic region. ${ }^{31}$ Previous studies on the genes involved in HD have focused on their roles in the vagal neural crest derived lineages. In fact many of the genes known to be involved
Abbreviations used in this paper: Dom, dominant megacolon; EDN3, endothelin 3; EDNRB, endothelin $B$ receptor; ENS, enteric nervous system; HD, Hirschsprung's disease; RT-PCR, reverse transcription-polymerase chain reaction. 
in HD are expressed in both the vagal and sacral neural crest regions during human embryonic development. ${ }^{32}{ }^{33}$ Therefore, it is likely that the genes which affect proliferation, migration, and differentiation of vagal neural crest and cause the development of HD will also have similar effects on the sacral neural crest.

Previous studies on the expression patterns of SOX10 have mainly focussed on fetal stage $^{19}{ }^{32}$ : the cellular distribution of SOX10 in postnatal tissues is not known. In order to understand the molecular basis underlying the pathology of $\mathrm{HD}$, we examined the cellular expression patterns of SOX10 in the colon of normal infants and non-syndromic HD patients. Our results on the cellular distribution of SOX10 suggests that this gene is expressed in cells that are of vagal as well as sacral neural crest origin and SOX10 may have an important role in the pathogenesis of HD.

\section{Methods}

TISSUES FROM PATIENTS

Colon tissues were obtained from 10 patients with HD during pull through operation. Age ranged from three months to four years. There were eight boys and two girls. Nine patients had classical short segment aganglionosis with rectal or rectosigmoid involvement and one patient had aganglionosis extending to the splenic flexure. There was no family history and no associated major anomalies or syndromes. After mobilisation of the hypertrophied and spastic colon, colon tissue samples were taken from the normal segment, transitional segment (hypoganglionic), and spastic segment (aganglionic). Tissues from normal controls were obtained from colon biopsies at gastrointestinal operation for age matched patients without HD $(n=10)$. These infants and children had anorectal anomalies (anorectal atresia $n=5$; anal atresia $n=2$; anterior ectopic anus $n=1$ ) and necrotising enterocolitis of the distal gut $(n=2)$ and underwent colostomy or closure of colostomy at unaffected parts of the colon.

Tissue samples were subdivided for fixation in $4 \%$ paraformaldehyde solution for histological sectioning, snap freezing in liquid nitrogen, and storage at $-80^{\circ} \mathrm{C}$ for molecular analysis.

\section{MUTATION DETECTION}

DNA was extracted from peripheral blood samples collected from patients using a QiaAmp blood kit (Qiagen, Valencia, California, USA). Six sets of primers were used to amplify SOX10 gene fragments covering exons 2 to 5 and intron-exon junctions by polymerase chain reaction (PCR). The PCR products were purified and the DNA sequences determined by an automated sequencer (ABI Prism 310) after cycle sequencing reactions (dRhodamine kit). All sequences were determined from both forward and reverse orientations. Mutations were confirmed using duplicate PCR templates.
RNA EXTRACTION AND REVERSE TRANSCRIPTION-POLYMERASE CHAIN REACTION (RT-PCR)

RNA samples were extracted from frozen tissues by the acid guanidinium-phenolchloroform extraction method. ${ }^{34}$ Reverse transcription (RT) was performed using the SuperScript kit (Life Technologies, Rockville, Maryland, USA) according to the manufacturer's instructions. For PCR reaction, the PCR reagent system kit from Life Technologies was used according to the manufacturer's instructions. The sequences of the $S O X 10$ primers were 5'-ATA CGA CAC TGT CCC GGC CCT AAA-3' and 5'-TTC TCC TCT GTC CAG CCT GTT CTC-3'. As a control $\beta$-actin primers were used and their sequences were 5'-ACT CTT CCA GCC TTC CTT CC-3' and 5'-CGT CAT ACT CCT GCT TGC TG-3'. The SOX10 and $\beta$-actin cDNAs were amplified in separate PCR reactions. Samples that lacked RT were also amplified to control for the presence of any contaminating genomic DNA.

IN SITU HYBRIDISATION

To prepare the $S O X 10$ probe, a $570 \mathrm{bp}$ fragment corresponding to the 3'-untranslated region was amplified using another set of primers (5'-TGC CCT GTT CCT TGC CCA CCT C-3' and 5'-GGC CTC TGT GCC AAC TCC TTC C-3') by RT-PCR using normal human infant colon RNA. The PCR product was cloned into pBluescript $\mathrm{KSII}^{+}$and the nucleotide sequence of the cloned product was confirmed by sequencing. Single stranded $\left[{ }^{35} \mathrm{~S}\right]-\mathrm{UTP}$ labelled sense and antisense riboprobes were generated from a linearised SOX10 clone. Synthesis of riboprobes, hybridisation, autoradiography, and histological staining were carried out as described previously. ${ }^{35}$ The isotope ${ }^{35} \mathrm{~S}$ is weak in decay energy and travels for only a short distance. We and others $^{35}$ have found in situ hybridisation using ${ }^{35} \mathrm{~S}$ labelled riboprobe to give very good resolution for localisation of gene transcripts, especially when used in combination with immunohistochemistry for adjacent sections (see below). Slides were exposed in the dark at $4^{\circ} \mathrm{C}$ for 21 days. Photomicrographs were taken on an Axioplan 2 microscope (Carl Zeiss) fitted with a Sony digital camera under dark field or bright field illumination.

\section{IMMUNOHISTOCHEMISTRY}

Paraffin sections were treated with $3 \% \mathrm{H}_{2} \mathrm{O}_{2}$ for 10 minutes to block endogenous peroxidase activity and with $10 \mathrm{mM}$ citrate buffer ( $\mathrm{pH}$ 6.0) at $85^{\circ} \mathrm{C}$ for 10 minutes for antigen retrieval. Sections were incubated with primary antibody (for S100: clone 4C4.9, NeoMarkers, Fremont, California, USA 1:100 dilution; for RET: anti-RET R5, Nagoya University, Nagoya, Japan 1:100 dilution) in phosphate buffered saline containing $0.1 \%$ Tween 20 and $10 \%$ horse serum at $4^{\circ} \mathrm{C}$ for 16 hours, and then with horseradish peroxidase conjugated secondary antibody at $37^{\circ} \mathrm{C}$ for one hour. Immunoreactivity was visualised using strepABComplex/HRP kit (Dako, Carpinteria, 
$\operatorname{SOX} 10$

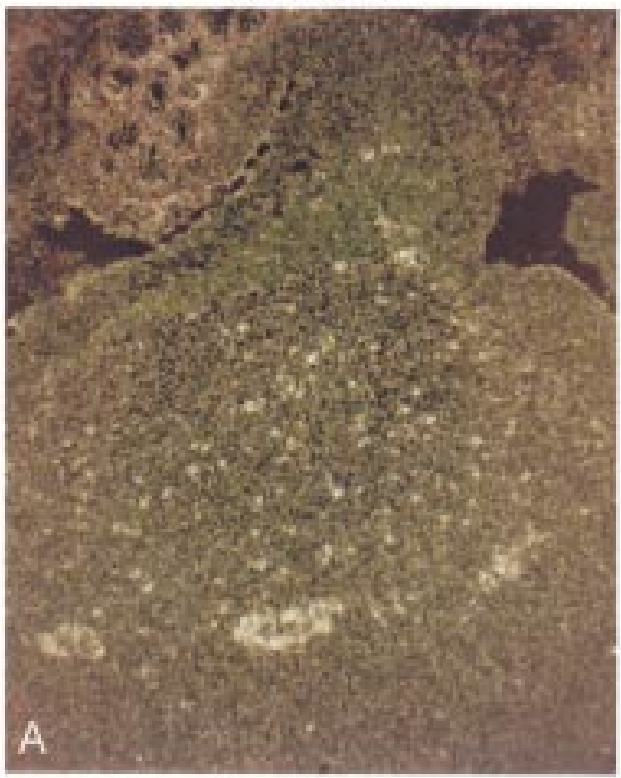

$\mathrm{S} 100$

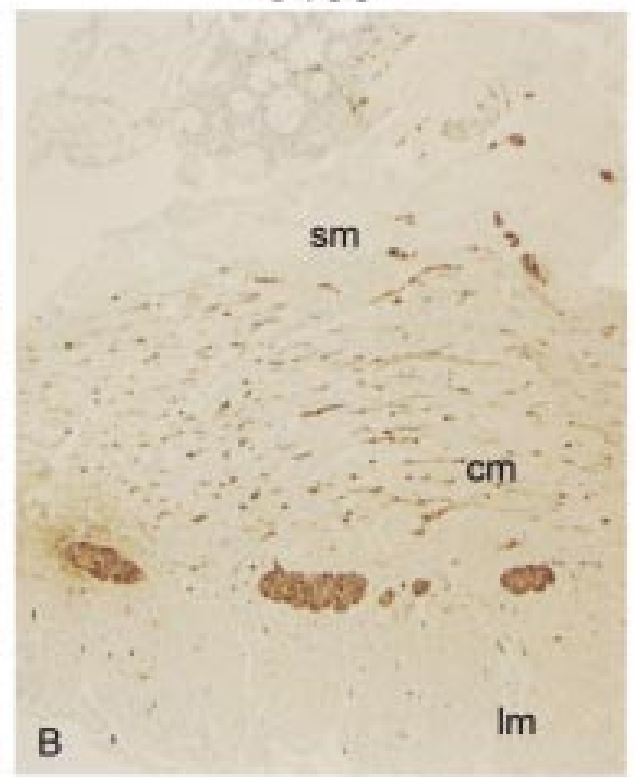

RET

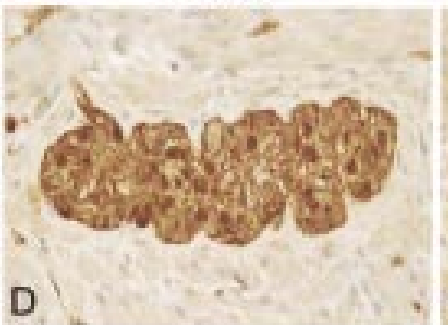

E
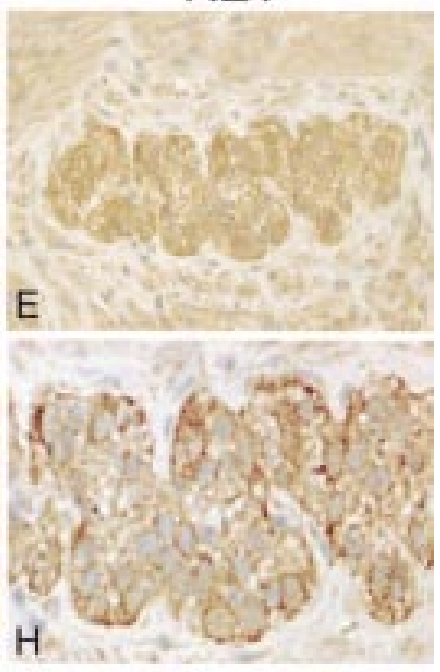

G
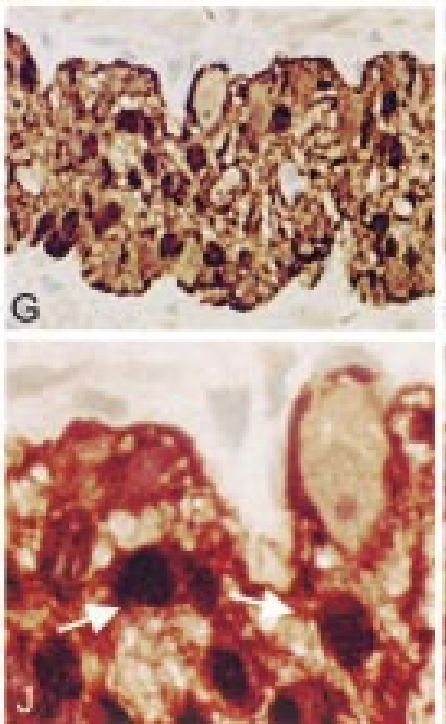

Figure 1 Expression of SOX10 in both neurones and glia of enteric plexuses in the colon of normal infants. (A) Dark field illumination of a cross section of normal colon showing strong in situ hybridisation signals in myenteric plexuses and in the nerves in the longitudinal and circular muscular layers. (B) Bright field illumination of an adjacent section showing S100 immunoreactivity in the SOX10 positive cells. (C) Dark field illumination and (F, I) bright field illumination of a myenteric plexus hybridised in situ with a SOX10 probe. $(D, G, \mathcal{F})$ Immunohistochemistry of an adjacent section with $S 100$ antibody showing that the glial cells present in the plexus are stained. $(E, H, K)$ Immunohistochemistry of another adjacent section with RET antibody showing that the neurones and nerves in the plexus are stained. For comparison, in I, $\mathcal{F}$, and $K$, the same glial cells are marked with arrows and the neurones are circled by broken lines. Both glial cells immunoreactive for $S 100$ and neurones immunoreactive for RET had positive SOX10 hybridisation signals. Original magnification: $(A, B) \times 100 ;(C, D, E) \times 400 ;(F, G, H) \times 500 ;(I, \mathcal{F}, K) \times 1000$. Sm, submucosa; cm, circular muscle; lm, longitudinal muscle. 
California, USA) and DAB (Sigma, St Louis, Missouri, USA). Sections were counterstained with haematoxylin, dehydrated, cleared in xylene, and mounted in DPX mountant (BDH, Poole, UK).

\section{Results}

We examined the presence and numbers of ganglionic plexuses in colon biopsies of HD patients. For each patient the tissue sample was divided into three segments designated aganglionic (spastic), hypoganglionic (transitional), and normoganglionic segments. For the aganglionic segments, we confirmed the absence of any ganglionic cells from the histological sections. We counted the number of ganglionic plexuses in the sections of hypoganglionic and normoganglionic colon segments and found fewer ganglia in the hypoganglionic than in normal segments. The difference however did not reach statistical significance. We performed sequence analysis on the SOX10 gene of HD patients. A polymorphic $\mathrm{A} \rightarrow \mathrm{G}$ change at nucleotide position 1876 (in exon 5, 475 nucleotides downstream of the stop codon) was detected in two patients. One of these two patients also had an additional silent mutation of CAC $\rightarrow$ CAT at codon 309. None of these nucleotide changes affect the SOX10 coding region and the other patients had normal SOX10 sequences.

EXPRESSION OF SOX10 IN THE COLON OF NORMAL INFANTS

It has previously been demonstrated by northern hybridisation that SOX10 is expressed in human colon. However, which cell types express SOX10 transcript is not yet known. We first examined bowel specimens from normal infants by in situ hybridisation. As shown in fig 1A, SOX10 mRNA was restricted to cells within the ganglionic plexuses. Clear punctate spots of SOX10 positive signals were also found in the nerve fibres of the muscular layers of the gut wall. By immunohistochemistry using an antibody against the glial cell marker $\mathrm{S} 100$ on adjacent sections, we found that the SOX10 hybridisation signals largely overlapped with cells which were immunoreactive for the $\$ 100$ antibody (fig 1B).

To further examine the cellular distribution of SOX10 in the neuronal plexuses of normal colon, we used anti-RET antibody which reacted with neuronal membrane bound RET receptor as a marker for enteric neurones and compared expression patterns of SOX10, $\mathrm{S} 100$, and RET. As shown in fig $1(\mathrm{C}-\mathrm{K})$, in the myenteric plexus a few RET immunoreactive cells were identified (fig $1 \mathrm{E}, \mathrm{H}, \mathrm{K}$ ) and the plexus contained a number of smaller S100 positive cells which were the enteric glia (fig $1 \mathrm{D}, \mathrm{G}, \mathrm{J})$. In the myenteric plexuses examined, SOX10 hybridisation signals were found in all cells in the ganglia, including neurones and glia (fig 1C, F, I). Therefore, in human colon, SOX10 expression is restricted to the ENS and is present in both neuronal and glial cells.
EXPRESSION OF SOX10 IN HD PATIENTS

We initially studied expression of SOX10 in HD patients by RT-PCR. As shown in fig 2, a DNA band of about 250 bp corresponding to a SOX10 fragment of expected size was detected in normal control colon tissues, and also in all three colon segment samples (normoganglionic, hypoganglionic, and aganglionic) obtained from HD patients. The control $\beta$-actin band of about $300 \mathrm{bp}$ was amplified from all cDNA samples. No DNA was amplified from the control samples which lacked RT, indicating that the amplified SOX 10 and $\beta$-actin fragments were derived from RNA. For all patients studied, we were able to detect expression of the SOX10 gene although at a lower level in the aganglionic segments (data not shown).

We performed semiquantitative PCR experiments using expression of the $\beta$-actin gene as a reference and we found that expression of SOX10 in the aganglionic segments was consistently lower than in hypoganglionic and normal segments (data not shown). The relative band intensity of SOX10 in 10 normal control samples was similar to that of the hypoganglionic and normal segments of patients (data not shown).

CELLULAR DISTRIBUTION OF SOX10 IN THE COLON OF HD PATIENTS

We further examined expression patterns of SOX10 in the colon of patients with HD. In the normoganglionic colon segments of HD patients, SOX10 mRNA was restricted to cells within the ganglionic plexuses (fig 3A, B). Clear punctate spots of SOX10 positive signals could also be found in the nerve fibres of the muscular layers of the gut wall (fig 3B). Similar results were obtained from hypoganglionic colon segments (data not shown). The pattern of SOX10 expression is similar to that in normal infants (fig 1). Interestingly, in the aganglionic segment of HD patients, despite the absence of functional neuronal cells, weak SOX10 hybridisation signals could be detected in clumps of cells which resembled ganglia but were in fact aggregates consisting of hypertrophied nerve trunks and glia (fig 3C, D). Detailed examination showed that SOX10

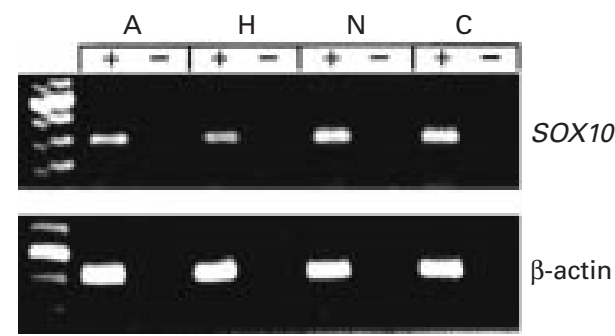

Figure 2 Expression of SOX10 in the aganglionic $(A)$, hypoganglionic $(H)$, and normoganglionic $(N)$ colon segments of a Hirschsprung's disease (HD) patient examined by reverse transcription-polymerase chain reaction (RT-PCR) in the presence (+) and absence (-) of reverse transcriptase. Expression of SOX10 in normal control colon tissue $(C)$ is also shown. The expected SOX10 fragment of about $250 \mathrm{bp}$ was amplified from normal control and HD patient samples. As a control the $\beta$-actin band of about $300 \mathrm{bp}$ was also amplified from all samples. No DNA fragment could be amplified from $c D N A$ samples that lacked reverse transcriptase ( - ) indicating that the amplified bands were derived from $m R N A$. 
expression could also be detected in the extrinsic nerves in the serosa (fig $3 \mathrm{E}-\mathrm{G}$ ) in addition to the hypertrophic nerves in the musculature (fig $3 \mathrm{H}-\mathrm{L}$ ) of the aganglionic segment. Immunohistochemistry of adjacent sections using antibodies against S100 (fig 3E, I) and RET (fig 3J)

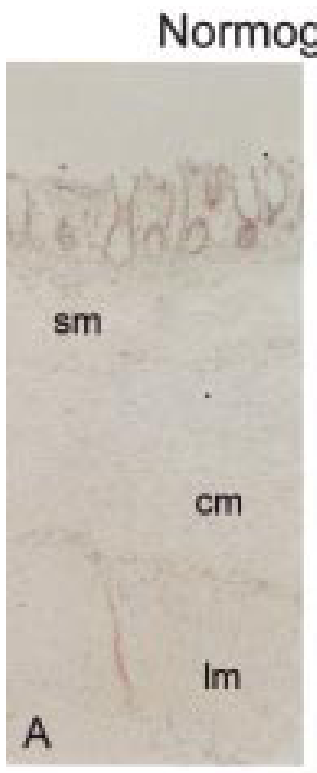

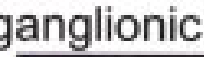

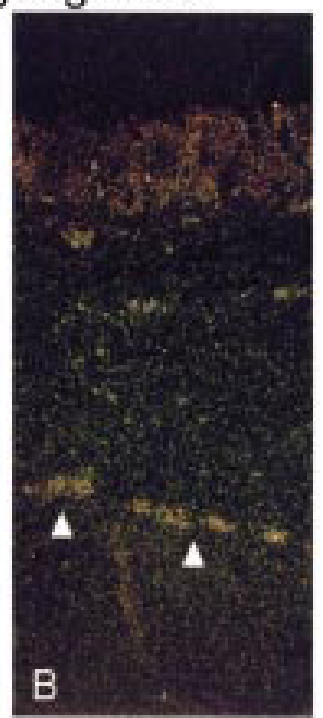

Aganglionic

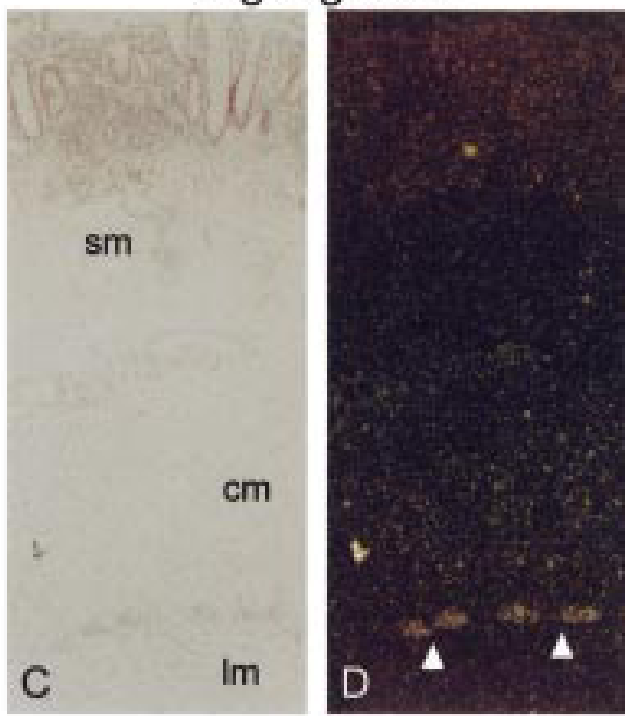

\section{Extrinsic nerve}

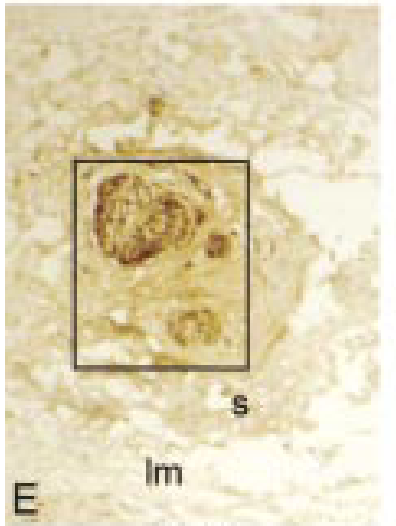

$\mathrm{S} 100$

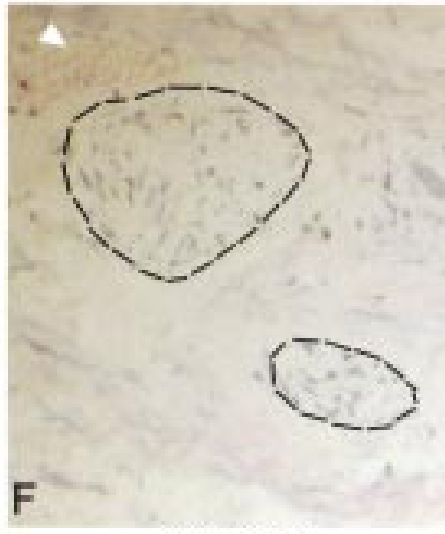

$\operatorname{sox} 10$

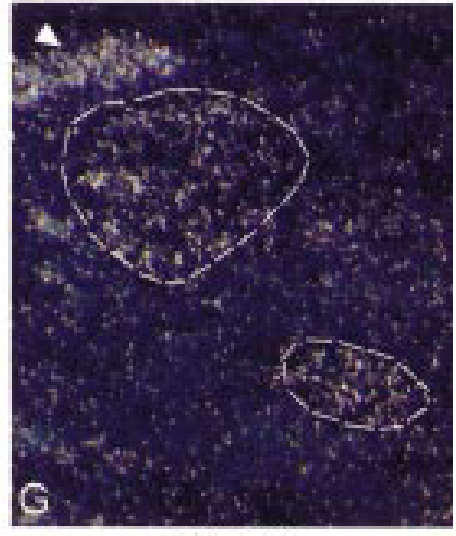

$\operatorname{SOX} 10$

Hypertrophic nerve
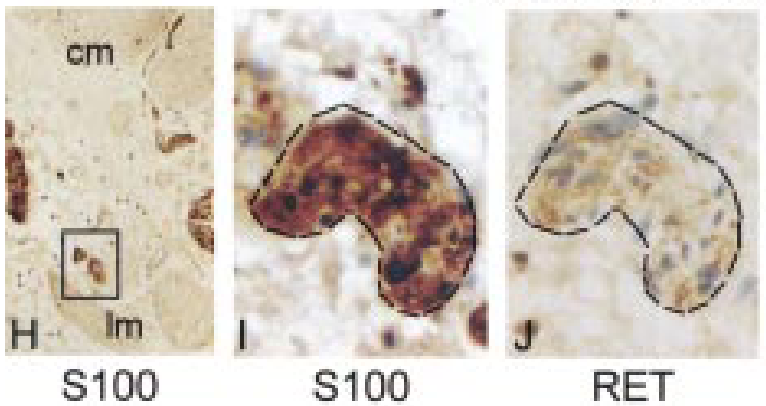

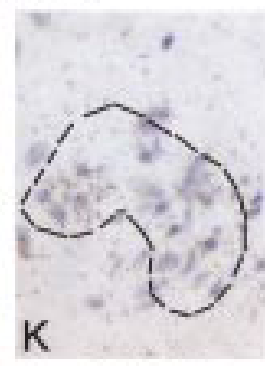

SOX10

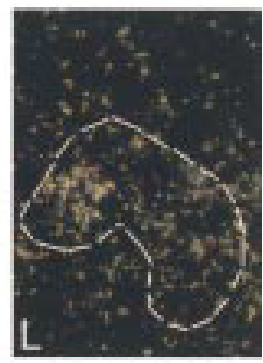

SOX10

Figure 3 Expression of SOX10 in the normoganglionic $(A, B)$ and aganglionic $(C-L)$ colon segments of a Hirschsprung's disease patient examined by in situ hybridisation. SOX10 is expressed in myenteric plexuses (arrowhead) and nerves in the circular and longitudinal muscles in the normoganglionic colon ( $A$, bright field; $B$, dark field). In the aganglionic colon, SOX10 is expressed in the hypertrophic nerve trunks and in nerves in the musculature $(C, K$, bright field; D, L, dark field). Immunohistochemistry on adjacent sections using $S 100(H, I)$ and RET (F) antibodies confirms that the hypertrophic nerves contain nerve fibres and glial cells but no neurones. The SOX10 positive signals are found in the glia and nerve bundles. In addition, SOX10 is also weakly expressed in extrinsic nerves (F, bright field; G, dark field; arrowhead, blood vessel) in the serosa. Immunohistochemistry on an adjacent section shows that the extrinsic nerve is immunoreactive for S100 antibody (E). Original magnification: $(A-D) \times 100 ;(E) \times 200 ;(F, G, I-L) \times 500 ;(H) \times 100$. $S m$, submucosa; cm, circular muscle; lm, longitudinal muscle; s, serosa. 
further supported the fact that the SOX10 hybridisation signals overlapped with the glial cells and nerve bundles. Our in situ hybridisation results confirmed our PCR analysis above in that SOX10 was indeed expressed in aganglionic bowel but at a reduced level compared with normal bowel segments.

\section{Discussion}

In developing mouse embryos, Sox10 is expressed in migrating neural crest and peripheral nervous system derivatives. At $12.5 \mathrm{dpc}$ Sox 10 can be detected in the gut in locations consistent with myenteric ganglia. ${ }^{21}{ }^{22}{ }^{36}$ At later stages of development Sox 10 is expressed in the peripheral nervous system in Schwann cells; in the central nervous system Sox10 transcripts are first detected in glial precursors and later in oligodendrocytes of the adult brain. This has led to the suggestion that in later development, Sox10 is important for defining and maintaining the identity of glial cells. ${ }^{36}$ In situ hybridisation has also been performed using early human embryos and similar expression patterns were found in humans as in mice. ${ }^{19}{ }^{32}$ However, specific expression of SOX10 in the ENS of postnatal tissue had not been examined previously.

We have studied expression of SOX10 in the colon of normal infants and HD patients. We showed that SOX10 was expressed in the colon tissues of normal control individuals, suggesting that SOX10 function is required not only during early fetal stages but also in the mature ENS of the gut after embryonic development. The expression pattern of SOX10 in the bowel segment is remarkably similar to that of neuronal nitric oxide synthase and nerve growth factor receptor. ${ }^{37}{ }^{38}$ Clear SOX10 expression was detected in the myenteric and submucosal plexuses, and around the nerve fibres in the longitudinal and circular muscular layers in normal bowel segments. Using glial and neuronal markers, S-100 and RET respectively on adjacent sections, we found that SOX10 positive signals overlapped with both glial and neuronal cells. Therefore, our results showed that both glial and neuronal cells in the ENS expressed SOX10. While in the peripheral and central nervous systems SOX10 may be required only in glial cells, ${ }^{36}$ in the infant ENS we have shown that SOX10 expression was not restricted to glia but was also found in neurones. Our results on the cellular distribution of SOX10 mRNA in enteric ganglia plexuses and nerves strongly support the fact that the SOX10 gene is involved in the normal development and functioning of the entire ENS.

The pathological characteristics of HD are the absence of ganglion cells in the myenteric and submucosal plexuses, and the presence of hypertrophied nerve trunks in the distal colon. The abnormal nerve bundles consist of cholinergic nerve fibres ${ }^{38} 40$; ; they are immunoreactive for an antibody against nerve growth factor receptor ${ }^{38}$ and they are believed to be extrinsic parasympathetic nerve fibres of sacral origin. ${ }^{31}{ }^{42}$ It is clear that the major cellular defect in HD is abnormal development of vagal neural crest derived intrinsic ganglia leading to a deficiency of ganglion cells to populate the distal colon. Normally, sacral neural crest cells also contribute to intrinsic ganglia in the distal colon and it is not clear why the sacral neural crest failed to compensate for the loss of ganglion cells in HD. In addition, their development in the gut, if at all, appeared to be grossly distorted with hypertrophied nerve fibres ending blindly in the gut wall. ${ }^{42}$ Our results showed that in HD patients, SOX10 expression in the distal colon was affected; there was a significant reduction in the level of SOX10 expression in the aganglionic bowel segment. Moderate levels of SOX10 expression were detected in the abnormal nerve trunks which occupy the space of the abnormal ganglia. In the aganglionic bowel segments of HD patients reduced SOX10 expression is likely to be due to the absence of intrinsic ganglion cells, which are normally SOX10 positive, in the affected distal colon. The residual weak SOX10 expression detected clearly came from extrinsic nerves originated from sources other than the vagal neural crest; the weak signals could probably also come from the glial cell population of the abnormal ganglia. We argue that in HD patients, SOX10 expressed in the affected sacral region may have abnormal interactions with neurotrophic factors ${ }^{38} 434$ or with acetylcholine receptor which could be transactivated by $\operatorname{SOX} 10,{ }^{45}$ and as a result stimulate the growth of cholinergic and other excitatory nerve fibres in the aganglionic bowel.

For the non-syndromic HD patients examined in this study, none had mutations in the coding region of the SOX10 gene except for two patients who had silent and polymorphic DNA changes in the SOX10 gene. These patients probably harboured different genetic alterations. It is therefore important to note that expression of the SOX10 gene was affected in all cases. This suggests that SOX10 may be a key molecule in the functioning of the enteric ganglia, and may be involved in different regulatory pathways and signalling cascades for the ENS. In the Dom mouse model, it was found that expression of ENDRB was reduced in Dom heterozygotes and was absent in Dom homozygous mutants, suggesting that the signalling pathway of EDN3 and its receptor ENDRB is linked, either directly or indirectly, with the activity of the transcription factor Sox 10 in mice. ${ }^{21}$ Mutations in the EDNRB and EDN3 genes have also been identified in Waardenburg-Hirschsprung patients, ${ }^{781011}$ suggesting a link between SOX10 and the endothelin signalling pathways. Recently, by cell transfection assays, it was demonstrated that $S O X 10$ together with $P A X 3$ could activate $M I T F$ and c-ret genes, which were crucial for melanocyte and enteric neurone development, respectively. ${ }^{46-50}$ Our SOX10 results would support the idea that the functions of SOX10 are associated with many of the candidate genes for HD and Waardenburg syndrome. 
Dr M Fu, a paediatric surgeon from Beijing's Children's Hospital, was a recipient of a GB Ong fellowship and Croucher Foundation Visitorship. The study was supported by a CRCG grant from the University of Hong Kong and a competitive earmarked research grant HKU 7358/00M from the Research Grant Council to Professor P Tam.

1 Fewtrell MS, Tam PKH, Thomson AH, et al. Hirschsprung's disease associated with a deletion of chromosome 10 (q11.2q21.2): a further link with the neurocristopathies? f Med Genet 1994;31:325-7.

2 Goyal R, Hirano I. Mechanisms of disease: The enteric nervous system. N Engl f Med 1996;334:1106-15.

3 Lyonnet S, Bolino A, Pelet A, et al. A gene for Hirschsprung disease maps to the proximal long arm of chromosome 10 . Nat Genet 1993;4:346-50.

4 Yin L, Barone V, Seri M, et al. Heterogeneity and low detection rate of RET mutations in Hirschsprung disease. Eur $\mathcal{F}$ Hum Genet 1992;2:272-80.

5 Salomon R, Attie T, Pelet A, et al. Germline mutations of the RET ligand GDNF are not sufficient to cause Hirschsprung disease. Nat Genet 1996;14:345-7.

6 Sancandi M, Ceccherini I, Costa M, et al. Incidence of RET mutations in patients with Hirschsprung's disease. $\mathcal{F}$ Pediatr mutations in patients

7 Edery P, Attie T, Amiel J, et al. Mutation of the endothelin-3 gene in the Waardenburg-Hirschsprung. Nat Genet 1996 12:442-4.

8 Hofstra R, Osinga J, Tan-Sindhunata G, et al. A homozygous mutation in the endothelin-3 gene associated with a combined Waardenburg type 2 and Hirschsprung phenotype (Shah-Waardenburg syndrome). Nat Genet 1996;12 445-7.

9 Puffenberger E, Hosada K, Washington S, et al. A missense mutation of the endothelin-B receptor gene in multigenic Hirschsprung's disease. Cell 1994;79:1257-66.

10 Attie T, Till M, Pelet A, et al. Mutation of the endothelin-receptor B gene in Waardenburg-Hirschsprung disease. Hum Mol Genet 1995;4:2407-9.

11 Amiel J, Attie T, Jan D, et al. Heterozygous endothelin receptor $\mathrm{B}(\mathrm{EDNRB})$ mutations in isolated Hirschsprung disease. Hum Mol Genet 1996;5:355-7.

12 Auricchio A, Casari G, Staiano A, et al. Endothelin-B receptor mutations in patients with isolated Hirschsprung disease from a non-inbred population. Hum Mol Genet disease from a

13 Kusafuka T, Wang Y, Puri P. Novel mutations of the endothelin-B receptor gene in isolated patients with Hirschsprung's disease. Hum Mol Genet 1996;5:347-9

14 Doray B, Salomon R, Amiel J, et al. Mutation of the RET ligand, neurturin, supports multigenic inheritance in Hirschsprung disease. Hum Mol Genet 1998;7:1449-52.

15 Bolk S, Pelet A, Hofstra R, et al. A human model for multigenic inheritance: Phenotypic expression in Hirschsprung disease requires both the RET gene and a new 9q31 locus. Proc Natl Acad Sci USA 2000;97:268-73.

16 Southard-Smith EM, Angrist M, Ellison JS, et al. The Sox10(Dom) mouse: modeling the genetic variation of Waardenburg-Shah (WS4) syndrome. Genome Res 1999;9: 215-25.

17 Pingault V, Bondurand N, Kuhlbrodt K, et al. SOX10 mutations in patients with Waardenburg-Hirschsprung mutations in patients with Waard
disease. Nat Genet 1998;18:171-3.

18 Inoue K, Tanabe Y, Lupski JR. Myelin deficiencies in both the central and the peripheral nervous systems associated
with a SOX10 mutation. Ann Neurol 1999;46:313-18.

19 Touraine R, Attie-Bitach T, Manceau E, et al. Neurological phenotype in Waardenburg syndrome type 4 correlates with novel SOX10 truncating mutations and expression in developing brain. Am F Hum Genet 2000;66:1496-503.

20 Wright EM, Snopek B, Koopman P. Seven new members of the Sox gene family expressed during mouse development. Nucleic Acids Res 1993;21:744.

21 Southard-Smith EM, Kos L, Pavan WJ. Sox10 mutation disrupts neural crest development in Dom Hirschsprung mouse model. Nat Genet 1998;18:60-4.

22 Herbarth B, Pingault V, Bondurand N, et al. Mutation of the Sry-related Sox10 gene in Dominant megacolon, a mouse model for human Hirschsprung disease. Proc Natl Acad Sci USA 1998;95:5161-5.

23 Pomeranz HD, Gershon MD. Colonization of the avian hindgut by cells derived from the sacral neural crest. Dev Biol 1990;137:378-94.

24 Pomeranz HD, Rothman TP, Gershon MD. Colonization of the post-umbilical bowel by cells derived from the sacral neural crest: direct tracing of cell migration using an intercalating probe and a replication-deficient retrovirus. Development 1991;111:647-55.

25 Serbedzija GN, Burgan S, Fraser SE, et al. Vital dye labelling demonstrates a sacral neural crest contribution to the enteric nervous system of chick and mouse embryos. Development 1991;111:857-66.

26 Gershon MD. Genes and lineages in the formation of the enteric nervous system. Curr Opin Neurobiol 1997;7:101-9.

27 Young HM, Hearn CJ, Ciampoli D, et al. A single rostrocaudal colonization of the rodent intestine by enteric neuron precursors is revealed by the expression of Phox $2 \mathrm{~b}$, Ret, and p75 and by explants grown under the kidney capsule or in organ culture. Dev Biol 1998;202:67-84.

28 Burns AJ, Le Douarin NM. The sacral neural crest contributes neurons and glia to the post-umbilical gut: spatiotemporal analysis of the development of the enteric nervous system. Development 1998;125:4335-47.

29 Burns AJ, Champeval D, Le Douarin NM. Sacral neural crest cells colonise aganglionic hindgut in vivo but fail to compensate for lack of enteric ganglia. Dev Biol 2000;219: $30-43$.

30 Erickson CA, Goins TL. Sacral neural crest cell migration to the gut is dependent upon the migratory environment and not cell-autonomous migratory properties. Dev Biol 2000;219:79-97.

31 Robertson K, Mason I, Hall S. Hirschsprung's disease: genetic mutations in mice and men. Gut 1997;41:436-41.

32 Bondurand N, Kobetz A, Pingault V, et al. Expression of the SOX10 gene during human development. FEBS Lett 1998; 432:168-72.

33 Brand M, Le Moullec J-M, Corvol P, et al. Ontogeny of endothelins-1 and -3, their receptors, and endothelin converting enzyme-1 in the early human embryo. 7 Clin Invest 1998;101:549-59.

34 Chomczynski P, Sacchi N. Single-step method of RNA isolation by acid guanidinium thiocyanate-phenol-chloroform extraction. Anal Biochem 1987;162:156-9.

35 Wilkinson DG, Nieto MA. Detection of messenger RNA by in situ hybridization to tissue sections and whole mounts. Methods Enzymol 1993;225:361-73.

36 Kuhlbrodt K, Herbarth B, Sock E, et al. Sox10, a novel transcriptional modulator in glial cells. $\mathcal{F}$ Neurosci 1998;18: 237-50.

37 Vanderwinden JM, De-Laet MH, Schiffmann SN, et al. Nitric oxide synthase distribution in the enteric nervous system of $\mathrm{H}$

38 Kobayashi H, O'Briain DS, Puri P. Nerve growth factor receptor immunostaining suggests an extrinsic origin for hypertrophic nerves in Hirschsprung's disease. Gut 1994; 35:1605-7.

39 Zhou Q, Wang S, Anderson DJ. Identification of a novel family of oligodendrocyte lineage-specific basic helix-loophelix transcription factors. Neuron 2000;25:343.

40 Frigo GM, Del Tacca M, Lecchini S, et al. Some observations on the intrinsic nervous mechanism in Hirschsprung's disease. Gut 1973;14:35-40.

41 Giaroni C, De Ponti F, Cosentino M, et al. Plasticity in the enteric nervous system. Gastroenterology 1999;117:143858.

42 Tam PKH, Boyd GP. Origin, course, and endings of abnormal enteric nerve fibres in Hirschsprung's disease defined by whole-mount immunohistochemistry. I Pediatr Surg 1990;25:457-61

43 Hoehner JC, Wester T, Pahlman S, et al. Alterations in neuotrophin and neurotrophin-receptor localization in Hirschsprung's disease. F Pediatr Surg 1996;31:1524-9.

44 Kuroda T, Ueda M, Nakano M, et al. Altered production of nerve growth factor in aganglionic intestines. $\mathcal{F}$ Pediatr Surg 1994;29:288-92.

45 Liu Q, Melnikova IN, Hu M, et al. Cell type-specific activation of neuronal nicotinic acetylcholine receptor subunit genes by Sox10. F Neurosci 1999;19:9747-55.

46 Verastegui C, Bille K, Ortonne JP, et al. Regulation of the microphthalmia-associated transcription factor gene by the Waardenburg syndrome type 4 gene, SOX10. F Biol Chem 2000;275:30757-60

47 Bondurand N, Pingault V, Goerich DE, et al. Interaction among SOX10, PAX3 and MITF, three genes altered in Waardenburg syndrome. Hum Mol Genet 2000;9:1907-17.

48 Lee M, Goodall J, Verastegui C, et al. Direct regulation of the microphthalmia promoter by Sox10 links mentation and deafness to WS2. F Biol Chem 2000;275: 37978-83.

49 Potterf SB, Furumura M, Dunn KJ, et al. Transcription factor hierarchy in Waardenburg syndrome: regulation of MITF expression by SOX10 and PAX3. Hum Genet 2000; 107:1-6.

50 Lang D, Chen F, Milewski R, et al. Pax3 is required for enteric ganglia formation and functions with Sox10 to modulate expression of c-ret. $\mathcal{F}$ Clin Invest 2000;106:963-71. 